\title{
Two Cases of Recurred Vertigo Following Perilymphatic Oval Window Fistula Repair
}

\author{
Hack Jung Kim ${ }^{(D)}$ and Won-Ho Chung ${ }^{(D)}$ \\ Department of Otorhinolaryngology-Head and Neck Surgery, Samsung Medical Center, \\ Sungkyunkwan University School of Medicine, Seoul, Korea
}

외림프 누공 폐쇄술 이후 어지럼이 재발한 환자 2예

김학중 - 정원호

성균관대학교 의과대학 삼성서울병원 이비인후과학교실

\author{
Received March 16, 2020 \\ Revised May 11, 2020 \\ Accepted May 13, 2020 \\ Address for correspondence \\ Won-Ho Chung, MD, PhD \\ Department of Otorhinolaryngology- \\ Head and Neck Surgery, \\ Samsung Medical Center, \\ Sungkyunkwan University \\ School of Medicine, 81 Irwon-ro, \\ Gangnam-gu, Seoul 06351, Korea \\ Tel $+82-2-3410-3579$ \\ Fax +82-2-3410-3879 \\ E-mail whchung12@gmail.com
}

The traumatic perilymphatic fistula (PLF) can cause hearing loss and disabling dizziness. These symptoms can be recovered spontaneously or by surgical repair. However, even after surgical PLF repair, patients may suffer from recurrent vestibular symptoms. The mechanism of recurrent symptoms is not clear. We experienced two patients who complained of recurrent vertigo long after surgical PLF repair. Both patients had severe sensorineural hearing loss and vestibular loss after head trauma. In both cases, after surgical repair, dizziness was completely subsided. However, several years later, the disabling vertigo recurred in both cases. The symptoms aggravated when position was changed. On the fistula test, the patients showed positive results. Surgical findings showed a fibrous band connection between the stapes and the tympanic membrane. After stapedectomy, the symptoms subsided. We discuss the two cases in regards to the possible mechanism of recurrent vertigo after surgical PLF repair.

Korean J Otorhinolaryngol-Head Neck Surg 2021;64(2):103-7

Key Words Floating stapes · Perilymphatic fistula $\cdot$ Vertigo.

\section{Introduction}

Perilymphatic fistulas (PLF) can be caused by various causes, including stapes surgery, head trauma, and inner ear erosion in chronic otitis media. ${ }^{1)}$ Symptoms of PLF are sensorineural hearing loss and dizziness.

Prognosis of hearing loss caused by PLF varies according to the time of occurrence and the type and severity of trauma. In the case of PLF due to barotrauma, the prognosis of hearing improvement is good if early surgical exploration is performed. ${ }^{2)}$ Dizziness caused by PLF may be a symptom of unilateral vestibular loss such as continuous whirling type

This is an Open Access article distributed under the terms of the Creative Commons Attribution Non-Commercial License (https://creativecommons.org/licenses/by-nc/4.0) which permits unrestricted non-commercial use, distribution, and reproduction in any medium, provided the original work is properly cited. vertigo and postural imbalance due to irreversible vestibular loss, or may also be symptom of positional dizziness. Atypical benign paroxysmal positional vertigo like positional nystagmus was found in the barotraumatic PLF. ${ }^{3)}$

Dizziness caused by PLF shows different prognosis according to the severity of vestibular loss and the characters of dizziness. If unilateral complete vestibular loss has occurred, vestibular rehabilitation can improve the vestibular symptoms.

In previous studies, dizziness caused by barotraumatic PLF often did not show unilateral vestibular loss, ${ }^{3)}$ and vestibular symptoms improved when surgery was performed on a suspected PLF.

We experienced two cases of recurred dizziness a few years after PLF repair in the traumatic PLF on the oval window (OW). 


\section{Case}

\section{Case 1. Hearing loss and repeated vertigo after surgery for left chronic otitis media}

A 30-year-old man visited our clinic for left hearing loss and dizziness that has occurred immediately after canal wall up mastoidetomy with partial ossicular replacement prosthesis due to left chronic otitis media a year ago at another hospital. In the pure tone audiometry (PTA), the left ear had profound hearing loss (Fig. 1A). The vertiginous dizziness was caused by touching the ear and position change, which lasted less than 5 minutes. In videonystagmograph (VNG), spontaneous nystagmus and gaze evoked nystagmus were not observed. In the fistula test, the left beating horizontal nystagmus was induced by positive pressure in the left ear canal. In the caloric test, $64 \%$ of canal paresis was recorded on the left. The temporal bone (TB) CT showed no fracture line in the otic capsule, but a pneumolabyrinth was identified, suggesting a OW fistula (Fig. 1B). Under the suspicion of PLF around the stapes, and reoperation was performed. At the time of operation, the subluxation of the stapes footplate was found. The partial ossicular replacement prosthesis was removed, and the fistula was repaired by applying soft tissue and the fibrin glue around the stapes footplate. Any prothesis was not placed over the stapes head. Vestibular symptoms improved immediately after surgery. Hearing was not improved.

Three years after the operation, similar characters of dizziness recurred. The vertiginous dizziness was caused by touching the ear and position change, which lasted less than 5 minutes. The fistula test was positive. Recurrence of PLF was suspected due to a positive fistula test, and exploration was done under the local anesthesia. Definite PLF was not found, but there was a fibrous band between the stapes head and the tympanic membrane (Fig. 1C). The patient com- plained of subjective severe dizziness when touching the stapes during operation. Because it seemed that the dizziness was caused by the excessive movement of the stapes, the stapedectomy was done. After stapedectomy, occlusion of OW was performed with a soft tissue. On top of that, a silastic sheet was placed over the $\mathrm{OW}$ to block pressure transmission from the outside. Immediately after surgery, the patient's dizziness disappears completely. He visited our clinic at 1 year postoperatively without any complaint about dizziness.

\section{Case 2. Hearing loss and repeated vertigo after blunt head trauma due to traffic accident}

A 45-year-old female visited the hospital for a hearing loss and dizziness after a traffic accident 8 years ago. When she visited the clinic for the first time, she complained of lightheadedness, and the dizzy spell was worsened when the position changed, or when touching the ears or laughing loudly. PTA showed the right profound hearing loss (Fig. 2A). On TBCT after traffic accident, pneumolabyrinth in the vestibule was found (Fig. 2B). In the caloric test, right canal paresis $(95 \%$ weaker) was recorded. Spontaneous nystagmus and gaze-evoked nystagmus were not observed in the VNG test and no significant nystagmus was observed in the positioning test, head shaking test. On fistula test, subjective dizziness was induced with upbeat nystagmus. Traumatic PLF was suspected and exploration was performed under local anesthesia. A fistula hole was identified in the OW with subluxation of the stapes (Fig. 2C). After removal of the incus, the fistula was repaired using soft tissue and cartilage. After surgery, the dizziness was completely improved. However, 8 years later, she revisited the clinic due to recurred dizziness. The symptom was induced when she put her finger in her ear or blow her nose. In the VNG, spontaneous nystagmus and gazeinduced nystagmus were not observed. In the fistula test,
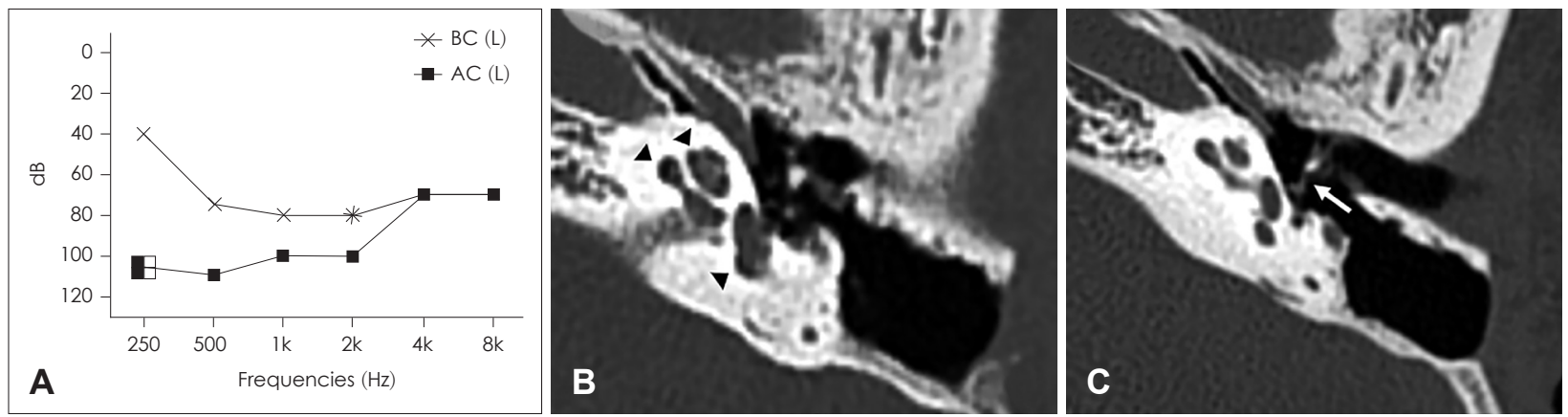

Fig. 1. Results of the pure tone audiometry and TBCT examination of case 1. Preoperative audiogram at 1 st operation showed a left profound hearing loss (A). Pneumolabyrinth in the vestibule (arrowhead) was detected on TBCT (B). Preoperative TBCT at 2nd operation showed a soft tissue connection (arrow) between the eardrum and the stapes head (C). AC: air conduction, BC: bone conduction. TBCT: temporal bone CT. 

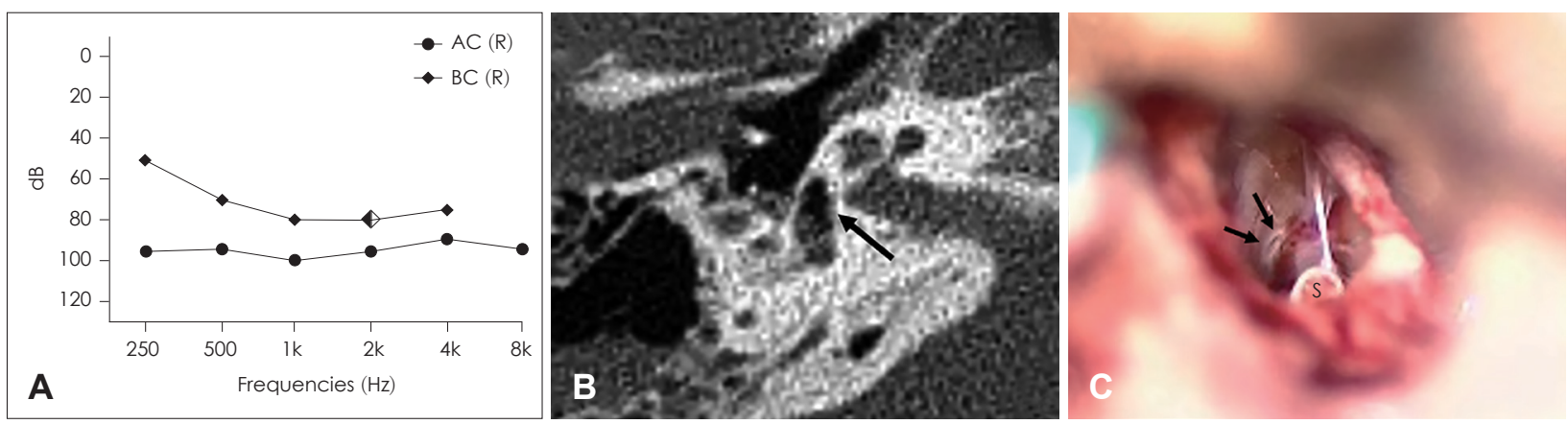

Fig. 2. Results of the pure tone audiometry and TBCT examination of case 2. Preoperative audiogram showed a right profound mixed hearing loss with a pure tone average (A). On TBCT after traffic accident, pneumolabyrinth in the vestibule (arrow) on the right axial TBCT view was found (B). In operative findings, fistular hole (arrows) was found around the stapes (C). AC: air conduction, BC: bone conduction, S: stapes, TBCT: temporal bone CT.

nystagmus was not observed, but subjective dizziness was complained. No abnormal findings were found on the TBCT. Exploration was performed under the suspicion of PLF under local anesthesia. In the surgical findings, the malleus handle were connected to the stapes head by the thick fibrous bands. Touching the stapes head during surgery induced subjective dizziness. Because it seemed that the dizziness was caused by the excessive movement of the stapes, the stapedectomy was done. After stapedectomy, we occluded the OW using soft tissue and cartilage. Thick silastic sheet (20/1000 inch) was placed over the OW. Dizziness disappeared completely immediately after surgery. Her last visit to our clinic was $t 2$ months after operation without any complaint about dizziness.

\section{Discussion}

We experienced two patients in similar findings who presented with positional dizziness and pressure induced dizziness after traumatic OW fistula. After fistular repair using soft tissue patch, the dizziness symptoms subsided, but the symptoms recurred a few years later. At revision surgery, even though actual fistula was not found, the stapes was connected with tympanic membrane by thick soft tissue band. When it was touched, the subjective dizzy spell was felt. After stapedectomy with soft tissue obliteration of OW, the dizzy spell subsided.

The two cases had several things in common. They were found to have OW fistula by iatrogenic injury (case 1) and blunt head trauma (case 2). Both cases had pneumovestibule after trauma on TBCT. At first operation, stapes subluxation (case 1) and definite fistular hole (case 2) was found. After repairing the fistula with soft tissue patching, the dizziness spell was subsided. At second operation after the dizzy symptoms recurred, no definite fistulas were not found, but thick fibrous band was connected between stapes head and tympanic membrane. They complained of dizziness when palpating the fibrous band or stapes head. After stapedectomy, the dizzy spells immediately subsided.

If the trauma causes dislocation and subluxation of the stapes, the PLF may occur at the OW, and pneumovestibule can be created. This causes sensorineural hearing loss and vestibular loss with whirling vertigo and unsteadiness because of severe damage of inner ear end organs. ${ }^{4)}$ Such hearing loss and vestibular loss is often irreversible, but subjective dizzy symptoms caused by unilateral vestibular loss tends to improve gradually by the vestibular compensation. However, if the PLF remains, postural dizziness or dizziness caused by middle ear pressure may persist.

In these cases, unilateral vestibular loss was initially proved in the caloric test, and the patient complained of subjective severe dizziness. These dizzy symptoms gradually improved as vestibular compensation proceeds over time. However, the patient was still complaining of positional dizziness if the fistula remains.

The mechanism of positional dizziness and pressure induced dizziness in PLF was not clear. Vestibular symptoms in chronic PLF have been assumed to be attributable to endolymphaic hydrops in the fistula ear. ${ }^{5)}$ On fistula test by canal pressure with a pneumatic otoscope (Hennebert's sign), nystagmus can be induced. In other animal study, artificial PLF could induce hydrops, rupture, and collapse of endolymphatic membrane in the inner ear. These pathologic change called "floating labyrinth" might be involved in prolonged subjective dizziness and nystagmus. ${ }^{6,7)}$

In our cases, iatrogenic and blunt trauma caused the disruption of annular ligament and subluxate the stapes. The stapes became floating, it can induce dizziness by stimulating the vestibuloocular reflex in the semicircular canal or 


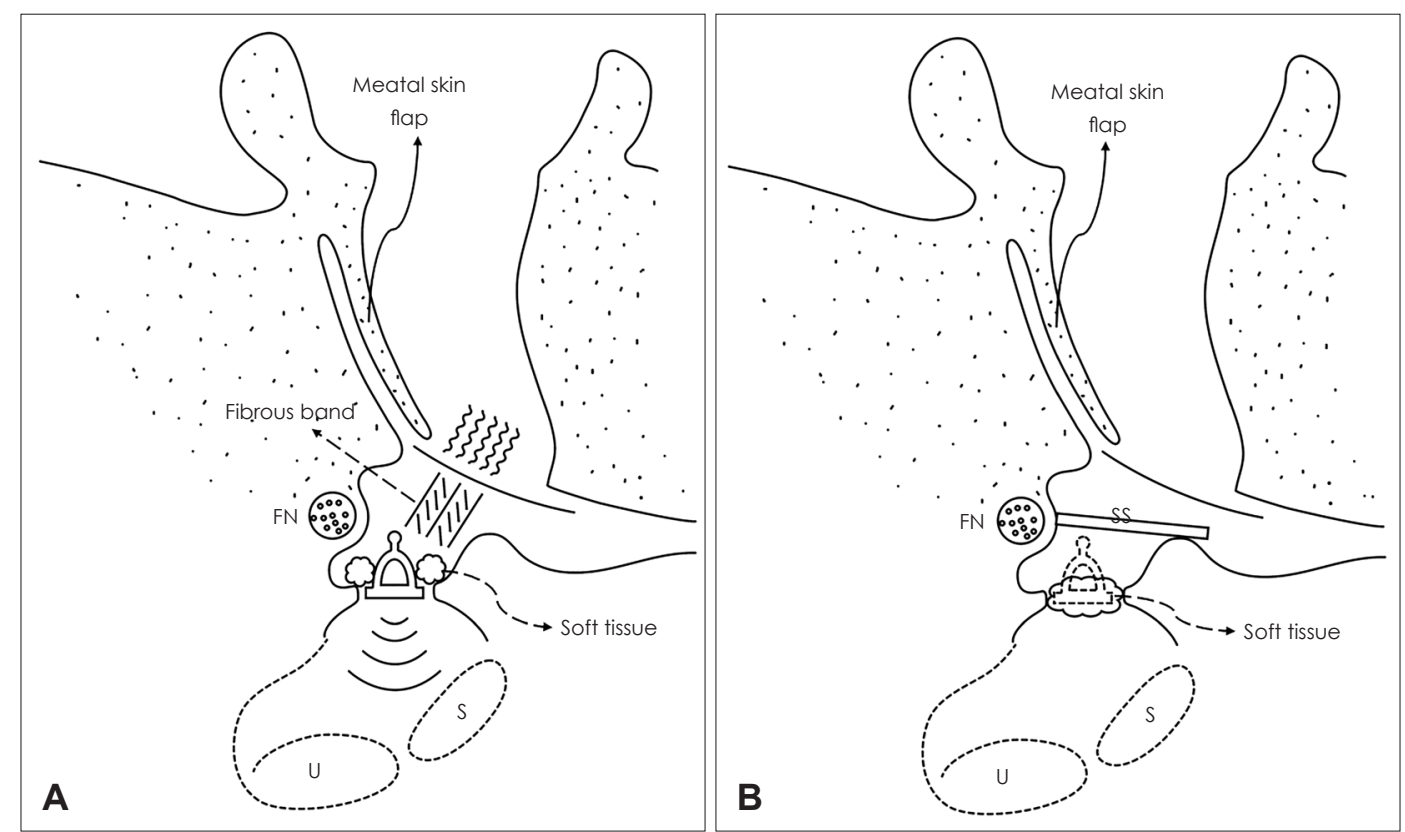

Fig. 3. The schematic operative findings at revision operation in both cases. The stapes was intact with any definte fistula. Thick fibrous band was found interconnecting between stapes head and tympanic membrane. The hypothesis of dizziness mechanism is that the movement of tympanic membrane was transmitted to the OW to stimulate otolithic organs. The environment in the vestibule has been changed (A). Operation procedures. To eliminate the interconnection between the otolithic organs and tympanic membrane, the stapes was removed and packed with soft tissue. To prevent regrowth of fibrous band, silastic sheet was placed over the OW (B). FN: facial nerve, S: saccule, U: utricle, SS: silastic sheet, OW: oval window.

the otolithic organs by pressure and positional change.

At the first operation, the stapes was not removed, and only the identified fistula was repaired with soft tissue, and the operation was completed. Dizziness improved immediately after the surgery. However, case 1 recurred after 1 year and 6 months, and case 2 after 8 years. In the second operation, there was a soft tissue connection by thick fibrous band between the stapes head and the tympanic membrane in both cases (Fig. 3A). The patient complained of severe dizziness when touching the fibrous band or the stapes. Therefore, it was confirmed that stapes movement caused dizziness, and this finding could explain the positive fistula test. Thus, after stapedectomy, the OW was occluded with soft tissue (Fig. 3B). Immediately after surgery, dizziness subsided completely in both patients.

The drawings showed the preoperative findings, and surgical procedure at revision operation (Fig. 3). My hypothesis for the mechanism of dizziness after OW fistula is the hypermobile stapes connected with inner ear endolymphatic membrane. Like Hennebert's sign, the stapes movement stimulated the vestibularocular reflex in the otolith organs. In normal inner ear, stapes movement is difficult to induce otolithic organs stimulation. This is because, in histological examination, the endolymphatic membrane of saccule and the stapes are separated from each other, and the perilymphatic fluid is present between them. ${ }^{8}$ However, in Meniere's disease, Hennebert's sign or Tullio phenomenon can occur. It means that endolymphatic membrane of the saccule contacts the stapes by endolymphatic hydrops, so the movement of the stapes by sound or pressure can stimulate the otolithic organs. ${ }^{9)}$ This causes the patient to complain of dizziness. Likewise, if the dislocation of stapes causing OW fistula changed the environment in the vestibule, such as hydrops, collapse, and rupture of endolymphatic membrane, or blood or inflammatory cells filling the vestibule, the movement of the stapes may stimulate the otolithic organs directed by interconnections between the stapes and the otolithic organs. ${ }^{6,10,11}$ In addition, if the stapes mobility has been changed due to the dislocation of the stapes, the otolithic ogans may be more easily stimulated by stapes movement (floating stapes).

Interesting findings in these cases were nystagmus direction in fistula tests. Both cases showed positive fistula tests. In case 1, the nystagmus direction was horizontal with fast component directed to lesion side. However, in case 2. The nystagmus direction was upward. These meant that the stimulating organs seemed to be otolith organs rather than semicircular canals.

From these cases, we found two important findings. First 
was that OW fistula due to stapes subluxation could induce persistent chronic dizziness. Therefore, if the patients complaining of persistent dizziness is suspected of OW fistula, surgical exploration should be considered. Second, even after the OW fistula was healed spontaneously or surgically repaired, disabling dizziness could persist. The underlying mechanism for this might be interconnection between stapes and otolithic organs to cause the Hennebert's sign due to unstable floating stapes or the environmental change in the vestibule (floating labryrinth) or both.

\section{Acknowledgments}

None.

\section{Author Contribution}

Writing — original draft: Hack Jung Kim. Writing_review \& editing: Won-Ho Chung.

\section{ORCIDs}

Won-Ho Chung

Hack Jung Kim

https://orcid.org/0000-0001-7157-9935

https://orcid.org/0000-0002-1294-9315

\section{REFERENCES}

1) Minor LB. Labyrinthine fistulae: Pathobiology and management.
Curr Opin Otolaryngol Head Neck Surg 2003;11(5):340-6.

2) Park GY, Byun H, Moon IJ, Hong SH, Cho YS, Chung WH. Effects of early surgical exploration in suspected barotraumatic perilymph fistulas. Clin Exp Otorhinolaryngol 2012;5(2):74-80.

3) Ahn J, Son SE, Choi JE, Cho YS, Chung WH. Surgical outcomes on hearing and vestibular symptoms in barotraumatic perilymphatic fistula. Otol Neurotol 2019;40(4):e356-63.

4) Choi JE, Moon IJ, Kim H, Lee K, Cho YS, Chung WH. Diagnostic criteria of barotraumatic perilymph fistula based on clinical manifestations. Acta Otolaryngol 2017;137(1):16-22.

5) Hornibrook J. A balance test for chronic perilymph fistula. Int J Otolaryngol 2012;2012:163691.

6) Nomura Y, Hara M, Young YH, Okuno T. Inner ear morphology of experimental perilymphatic fistula. Am J Otol 1992;13(1):32-7.

7) Nomura $Y$, Hara M, Funai H, Okuno T. Endolymphatic hydrops in perilymphatic fistula. Acta Otolaryngol 1987;103(5-6):469-76.

8) Backous DD, Minor LB, Aboujaoude ES, Nager GT. Relationship of the utriculus and sacculus to the stapes footplate: Anatomic implications for sound-and/or pressure-induced otolith activation. Ann Otol Rhinol Laryngol 1999;108(6):548-53.

9) Okuno T, Sando I. Localization, frequency, and severity of endolymphatic hydrops and the pathology of the labyrinthine membrane in Menière's disease. Ann Otol Rhinol Laryngol 1987;96(4):438-45.

10) Nadol JB Jr. Positive Hennebert's sign in Meniere's disease. Arch Otolaryngol 1977;103(9):524-30.

11) Paparella MM. Interactive inner-ear/middle-ear disease, including perilymphatic fistula. Acta Otolaryngol Suppl 1991;485:36-45. 